\title{
Experimental Analysis of All Optical NOT gate and Latch based on an Externally Clamped Semiconductor Optical Amplifier
}

\author{
Sergio L. Stevan Jr and António L. J. Teixeira
}

\begin{abstract}
An all-optical NOT gate is experimentally demonstrated recurring to external gain clamping of a semiconductor optical amplifier. Latch principle is subsequently demonstrated with the aid of an optical ring laser, using off-theshelf devices.
\end{abstract}

Index Terms - NOT Gate, SOA, Optical Latch

\section{INTRODUCTION}

$\mathrm{S}$ EMICONDUCTOR Optical Amplifiers (SOA) are alloptical amplification devices that have been widely used in different applications. In special, they can be used as optical gates and other all-optical logical devices in very simple ways, like gain compression [1]. However, the speed and relation between the 'on' and 'off' state in the latter strategy is limited. Therefore, other processes, which could help the execution of higher speed switching, should be employed [2], and the integration is fundamental, that results at several costs [3].

Gain clamping is frequently used for improving gain linearity and saturation power [4]. Gain clamping is automatic gain control scheme obtained inducing a lasing effect though a resonant cavity formed by a pair of reflective devices, e.g. Fiber Bragg gratings at wavelength close to the range of signal wavelength. This laser effect will use the excess gain available when channel power is varied, maintaining the gain constant over a larger power range. The internal laser power was used as a reservoir of optical energy that extends the linear gain range before gain compression. When the laser energy is consumed laser action turns off [5]. With an adequate project of the reflective devices and their optimum couple at the SOA can be obtained a NOT gate of a signal extern with the control of the internal laser power.

The authors would like to acknowledge the NoE E1+, CONPAC (POSC/EEA-CPS/61714/2004), and FCT (SFRH/BD/29315/2006, BDE/15543/2005) and ALBAN (E03D03894BR) fellowships.

Sergio L. Stevan Jr. is with the Federal University of Technology - Paraná (UTFPR-PG). Monteiro Lobato Ave., km 04. CEP: 84016-210, Ponta Grossa/Brazil, (e-mail: sstevanjr@utfpr.edu.br).

António L. J. Teixeira is with Institute of Telecommunications, Aveiro Pole, 3810-193, Aveiro - Portugal, and with Department of Electronic, Telecommunications and Informatics, University of Aveiro, 3810-193 Aveiro, Portugal (e-mail: teixeira@ua.pt)
This paper presents an all-optical latch principle, based on Gain-Clamped SOA (GC-SOA) using commercial off-theshelf devices of low cost [5]. Due to lack of available devices in the laboratory, in special, the existence of only a pair of nanopositioners, a SOA ring laser was build and it was coupled after the GC-SOA to demonstrate the digital alloptical latch principle.

\section{SETUP AND RESUltS}

Figure 1 a) shows the setup of the optical NOT gate based on Gain Clamped-SOA, using off-the-shelf devices. The active device is a commercial C-band Booster Optical Amplifier Chip, with several specific characteristics, in special, the angles of coupling, where the lateral beam exit angle are $19.5^{\circ}$ [6]. The gain chip was fixed in a nanopositioner and a laser current controller is set to $280 \mathrm{~mA}$. The pair of FBG, centered at $1544.9 \mathrm{~nm}$ were recorded near of the end of the fibers, and carefully aligned with nanopositioners as close as possible of the SOA to the light to be coupled at the two sides, as shown in the schematic of the figure $1 \mathrm{~b}$ ). This process is critical to couple the maximum signal on the fibers, and the fiber's signal to SOA, and consequently the losses due reflections, attenuations and performance of the GC-SOA is very dependent of this couple.

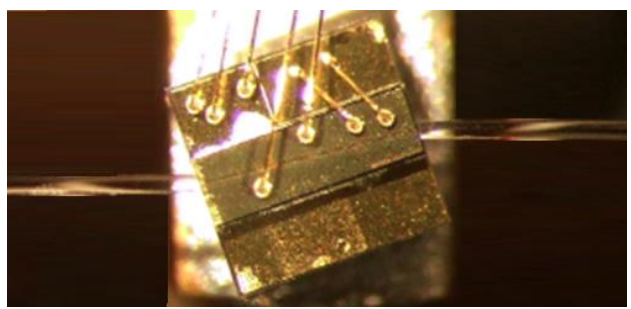

(a)

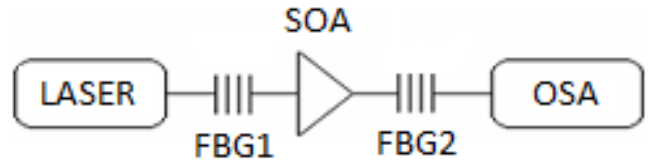

(b)

Figure 1: (a) NOT gate setup: Optical SOA chip aligned with related FBGs; and (b) simply schematic diagram of the gate. 
Figure 2 presents the reflectivity spectrums of the pair of FBG used. These gratings were used with the central wavelength closest available, about $1544,97 \mathrm{~nm}$, and the $3 \mathrm{~dB}$ bandwidth are approximately $0,090 \mathrm{~nm}$ to FBG1 and $0,120 \mathrm{~nm}$ to FBG2, with reflectivities about $50 \%$.

In order to test the saturation effect of the internal laser, showing therefore its erasure, an external laser at $1552 \mathrm{~nm}$ was coupled in one of the fibers and your power was varied from $7 \mathrm{dBm}$ to $+4 \mathrm{dBm}$. In the other side, an Optical Spectrum Analyser (OSA) was coupled to verify the evolution of the external and the internal laser powers. With this measurement, one can notice that there is an input Power range where an increase of $2 \mathrm{~dB}$, from $0 \mathrm{dBm}$ to $2 \mathrm{dBm}$, in the input laser power resulted in an extinction of about $20 \mathrm{~dB}$ of the internal laser. This behaviour can be seen in Figure 3, where the output peak power of the external laser (dotted line, at $1552 \mathrm{~nm}$ ) and the internal laser (solid line, at $1544 \mathrm{~nm}$ ) varying as a function of the change in input power of external laser. The OSA resolution used was set at $0.2 \mathrm{~nm}$.

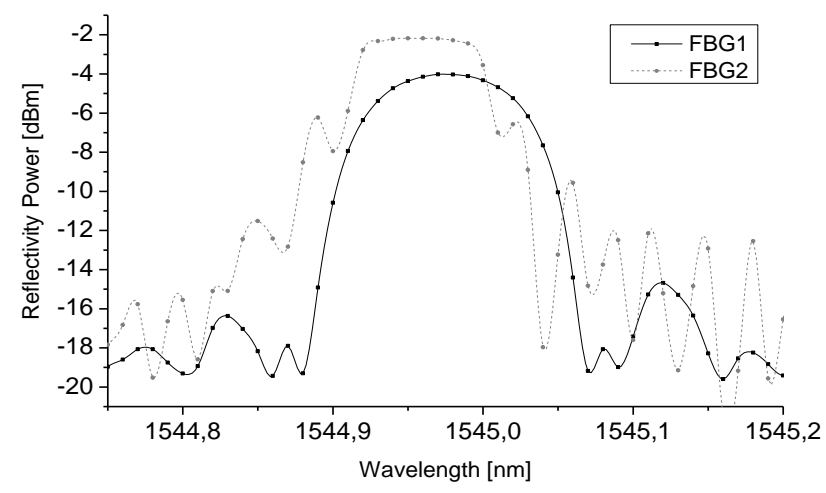

Figure 2: Spectrum of the pair of FBG used on design of Optical NOT Gate setup with SOA.

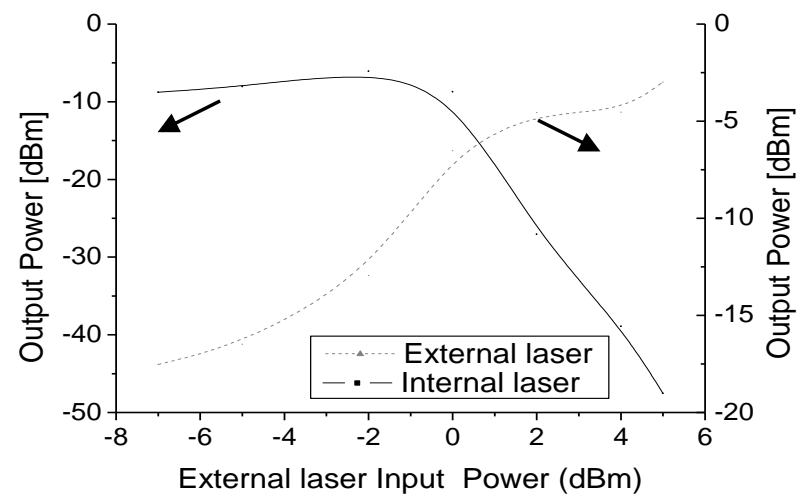

Figure 3: Output powers of the external and internal laser as a function of the external laser subsystem input Power.

Due of laboratory limitations (only a pair of nanopositioners was available), a SOA ring based laser was by us designed using isolators, a SOA and an optical filter available based on FBG centered at $1550 \mathrm{~nm}$, shown in figure 4 . The bandwidth of the filter used is about $0,5 \mathrm{~nm}$. The ring laser has about 3 meters of the fiber, resulting in a large resonant cavity and consequently slow response time laser [7]. Figure 5 presents the internal laser power control based on the power variation of external laser

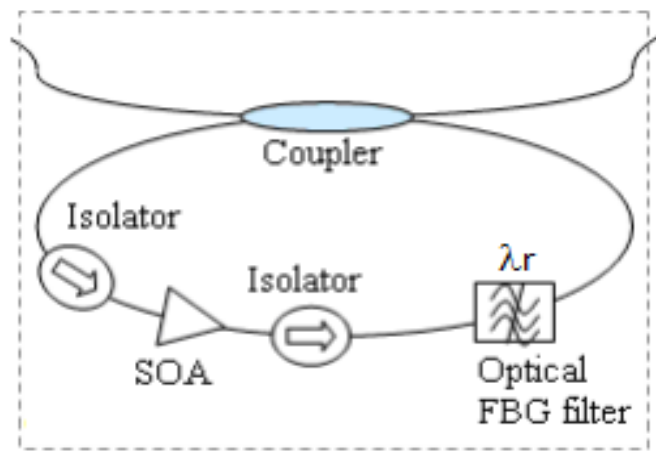

Figure 4: laser ring SOA setup.

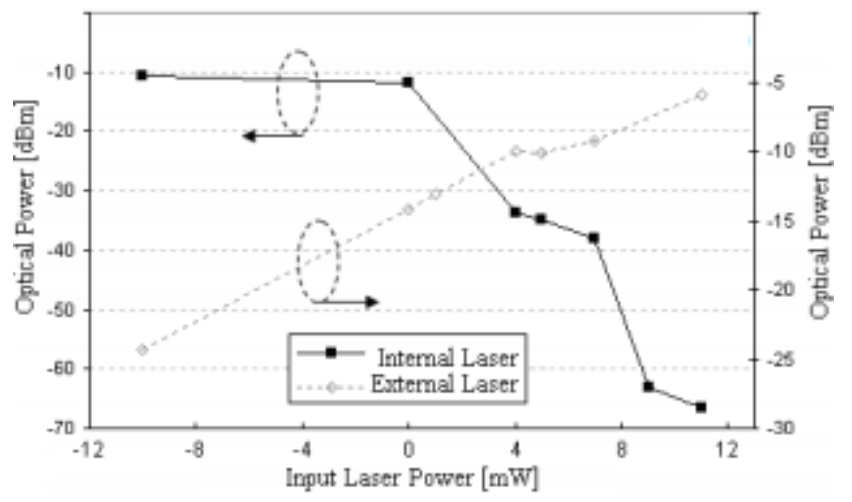

Figure 5: Output powers of the external and internal laser as a function of the external laser subsystem input power on laser ring SOA.

In a second moment, the SOA ring laser had been coupled to the GC-SOA to demonstrate the optical latch operation, as shown in the figure 4 (a). In this new setup, a power variable external laser (PE) was set at $1542 \mathrm{~nm}$ and coupled to the input of the GC-SOA (with internal laser at wavelength $\lambda w$ ). On the other side of the GC-SOA, a FBG with central wavelength marked as $\lambda$ pe (same of the PE) reflects PE and isolating the next stage. This FBG ( $\lambda$ pe) is coupled to the input of a ring laser SOA (based in an optical filter of wavelength $\lambda \mathrm{r}$ ), and the output is connected in an optical spectrum analyzer (OSA), as shown in Figure 6.

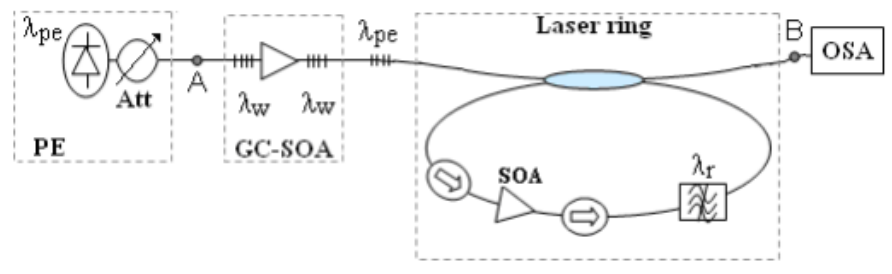

Figure 6: Experimental setup to demonstrate the digital all-optical latch principle 
The experimental results are presented in Figure 7, where, in figure 7 (a) are presented the OSA optical spectrum, when the external laser ' $\lambda$ pe' is on the state 'off', the internal laser of the wafer gain semiconductor ' $\lambda \mathrm{w}$ ' is on the state 'on' erasing the internal ring laser ' $\lambda \mathrm{r}$ '. When the external laser ' $\lambda$ pe' is on state 'on', it erases the laser ' $\lambda w$ ' and consequently brings to the 'on' state laser ' $\lambda r$ ', as shown in figure 7 (b). This stage should be kept stable even after turning off the laser ' $\lambda$ pe', as shown in figure 7 (c).
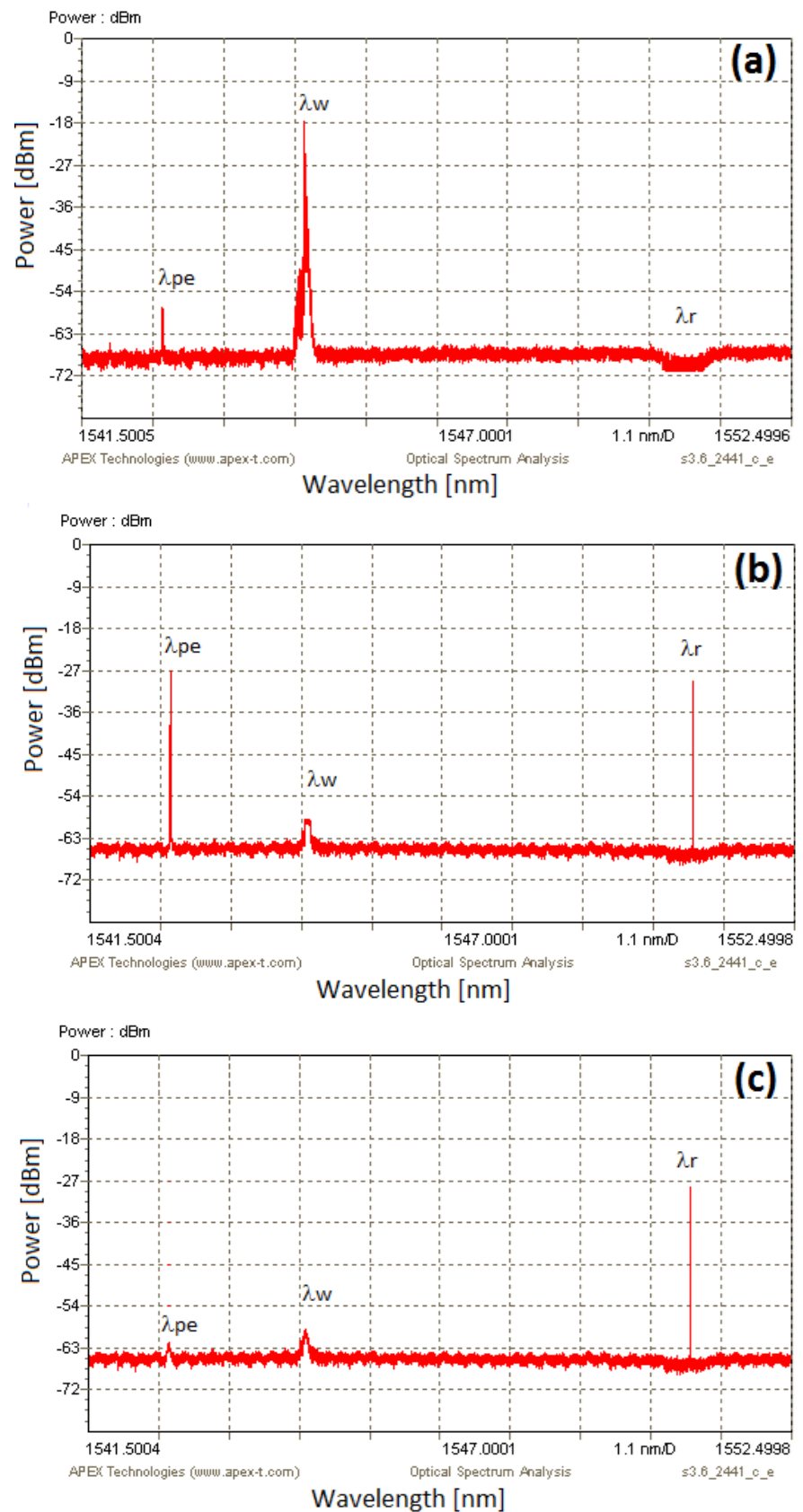

Figure 7: Demonstration of the digital all-optical latch principle, where: (a) Spectra of internal lasers $\lambda w$ 'on' and $\lambda r$ 'off' a initial state with External laser $\lambda$ pe 'off'; (b) Spectra induced of internal lasers $\lambda \mathrm{w}$ 'off' and $\lambda \mathrm{r}$ 'on' after External laser $\lambda$ pe changes to state 'on'; and, (c) Spectra of internal lasers $\lambda w$ and $\lambda$ pe without state changes after the External laser $\lambda$ pe 'off'.
To illustrate the response time of the proposed optical device based on off-the-shelf components, we present in Figure 8, the optical NOT gate based on GC-SOA (from Figure 1), operating at 1.244 GHz and in Figure 9, operating at $5.33 \mathrm{GHz}$ to demonstrate the possible rate of working of the proposed optical device.

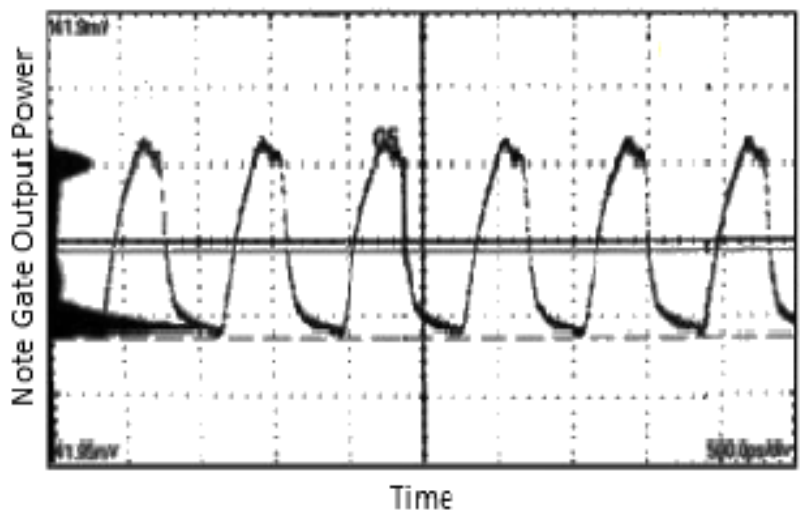

Figure 8 - Response time of the NOT gate to $1.244 \mathrm{GHz}$ with about $5.5 \mathrm{~dB}$ of ratio extinction

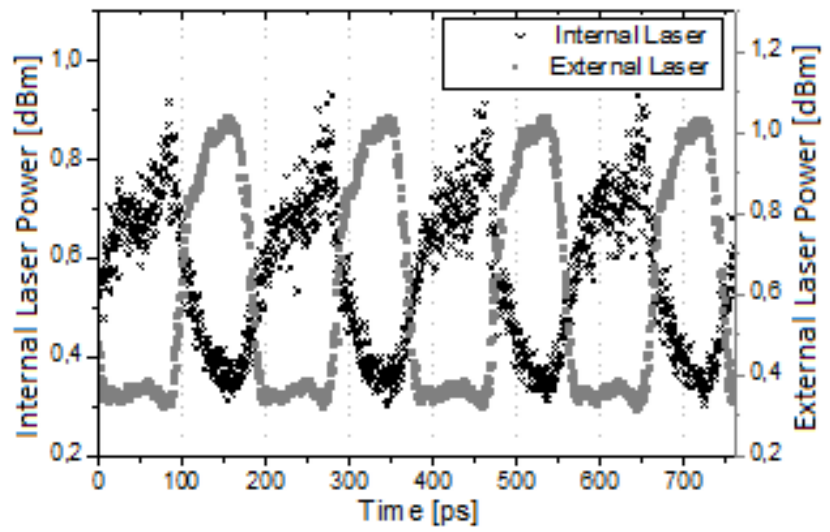

Figure 9 - Response time of the NOT gate to $5.33 \mathrm{GHz}$, with about $3.5 \mathrm{~dB}$ of ration extinction of internal laser.

In Figure 8 is presented the optical behaviour of the internal laser modulated at $1.244 \mathrm{GHz}$, witch improve an extinction rate of $5.5 \mathrm{~dB}$. The Figure 9 shows the external laser input modulated at $5.33 \mathrm{GHz}$ and the behavior of the internal laser, with extinction rate is about $3.5 \mathrm{~dB}$. This last case represents the maximum rate admitted in this experience. However, this obtained modulation rate can be extended optimizing the characteristics of the experience, as optical alignment and the optical coupling quality on the stages of the setup.

\section{CONCLUSION}

We reported experimental results from a Gain-clamped Semiconductor Optical Amplifier using discrete off-the-shelf components. The results showed that a small increase in the external controlling laser power (about $2 \mathrm{~dB}$ ), when near the gain saturation range of the gain chip, results in a quick erasure of the internal laser (about $30 \mathrm{~dB}$ ), resulting in a good 
extinction NOT gate. The latch principle has been demonstrated partially using an additional SOA ring laser. The response time of the latch is the same order of the NOT gate. We obtain the maximum internal' toggling rate of $5.33 \mathrm{GHz}$ with the extinction rate of about $3.5 \mathrm{~dB}$. The demonstration of the logic principles using commercial off-the-shelf components confirm the purpose to take for simple and low cost optical processors, where the couple optimization and incremental laboratory facilities available rightly will reach faster results.

\section{REFERENCES}

[1] M. J. Connelly ,'Semiconductor Amplifiers and Their Applications' (Invited paper),3rd Spanish Meeting of Optoelectronics, OPTOEL'03, Madrid, Spain, 14-16 July 2003.

[2] L. Q. Guo, and M. J. Connelly, "All-optical AND gate using nonlinear polarization rotation in a bulk semiconductor optical amplifier," in Technical Digest: Optical Amplifiers and Their Applications 2005 (The Optical Society of America, Washington, DC, 2005), Pres. no.: SuB9.

[3] E. Kehayas, J. Seoane, Y. Liu, J. M. Martinez, J. Herrera, P. V. HolmNielsen, S. Zhang, R. McDougall, G. Maxwell, F. Ramos, J. Marti, H. J. S. Dorren, P. Jeppesen, and H. Avramopoulos, "All-optical network subsystems using integrated soa-based optical gates and flip-flops for label-swapped networks," IEEE Photon. Technol. Lett. 18, 1750-1752, (2006)

[4] Silveira, T.; Teixeira, A.; Ferreira, Ana; B., G. Tosi; Forin, D.; Stevan, S.; Monteiro, P., "All-Optical Signal Processing Using Gain Clamped Semiconductor Optical Amplifiers", International Conference on Transparent Optical Networks, 2007. ICTON0, 3, 307-310, (2007)

[5] S. Stevan Jr., M. C. Fugihara, G. Tosi Bellefi, P. André,T. Silveira, R. Nogueira, A. Teixeira, A. Pohl, "Experimental Analysis of All Optical gate based in Gain Clamping Semiconductor Gain Chip", CLEO /EuropeIQEC 2007, Germany, (2007)

[6] Covega - BOA1007 - http://www.covega.com/products/pdfs/ BOA\%201007\%20Rev\%20C.pdf - datasheet disponible at 2010 January.
[7] S. Zhang, Z. Li, Y. Liu, G. Khoe, and H. Dorren, "Optical shift register based on an optical flip-flop memory with a single active element," Opt. Express 13, 9708-9713 (2005).

Sergio L. Stevan Jr was born in Brazil. He receivd the electrical engineering degree from Federal University of Paraná, in 1999; the Master's degree in Science from Technological Federal University of Paraná in 2001; and electronics engineering degree in electrical engineering all from the University of Aveiro, Aveiro, Portugal, in 2011.

Since 2011, he has been an Associate Professor at the Department of Electronics of Technological Federal University of Paraná - Ponta Grossa/PR Pole. He is also editor of the Brazilian Journal of Instrumentation and Control. His actual researches topics include optical devices and electronic instrumentation (with focus in electronic automotive development, biomedical applications and industrial automation).

Antonio Teixeira was born in Portugal. He received the electronics engineering degree and telecommunications and the Ph.D. degree in electrical engineering all from the University of Aveiro, Aveiro, Portugal, in 1994 and 1999, respectively.

$\mathrm{He}$ is currently an Associate Professor at the Department of Electronics, Telecommunications and Informatics, the University of Aveiro and Researcher at the Instituto de Telecomunicações - Aveiro. His re-search interests include optical networks, mostly access networks, and all-optical routing and switching technologies.

Dr. Teixeira is a member of OSA, TPC of major conferences like OFC and ECOC, and several other conferences like ICTON, Networks, GLOBECOM. $\mathrm{He}$ has more than 100 peer reviewed journal papers, and more than 300 conference papers. He is an Editor of several books and optics express. He has tutored more than 60 students (more than $15 \mathrm{PhD}$ and post doc). He is also with NSN as a Senior Specialist on access networks in IEEE standards association and FSAN/ITU-T Q2 and Q6. 